Spectral study of the HESS J1745-290 gamma-ray source as dark matter signal

This article has been downloaded from IOPscience. Please scroll down to see the full text article.

JCAP04(2013)051

(http://iopscience.iop.org/1475-7516/2013/04/051)

View the table of contents for this issue, or go to the journal homepage for more

Download details:

IP Address: 147.96.14.16

The article was downloaded on 26/06/2013 at 19:42

Please note that terms and conditions apply. 


\title{
Spectral study of the HESS J1745-290 gamma-ray source as dark matter signal
}

\author{
J.A.R. Cembranos, V. Gammaldi and A.L. Maroto \\ Departamento de Física Teórica I, Universidad Complutense de Madrid, \\ E-28040 Madrid, Spain \\ E-mail: cembra@fis.ucm.es, vivigamm@ucm.es, maroto@fis.ucm.es
}

Received March 12, 2013

Accepted April 16, 2013

Published April 30, 2013

\begin{abstract}
We study the main spectral features of the gamma-ray fluxes observed by the High Energy Stereoscopic System (HESS) from the J1745-290 Galactic Center source during the years 2004, 2005 and 2006. In particular, we show that these data are well fitted as the secondary gamma-rays photons generated from dark matter annihilating into Standard Model particles in combination with a simple power law background. We present explicit analyses for annihilation in a single standard model particle-antiparticle pair. In this case, the best fits are obtained for the $u \bar{u}$ and $d \bar{d}$ quark channels and for the $W^{+} W^{-}$and $Z Z$ gauge bosons, with background spectral index compatible with the Fermi-Large Area Telescope (LAT) data from the same region. The fits return a heavy WIMP, with a mass above $\sim 10 \mathrm{TeV}$, but well below the unitarity limit for thermal relic annihilation.
\end{abstract}

Keywords: dark matter theory, dark matter experiments, particle physics - cosmology connection, gamma ray experiments

ArXiv ePrint: $1302.6871 v 2$ 


\section{Contents}

1 Introduction 1

2 HESS J1745-290 data 2

3 Gamma-rays from dark matter annihilation 4

4 Single-channel fits $\quad 5$

5 FERMI 1FGL J1745.6-2900 data 6

6 Conclusions $\quad 9$

$\begin{array}{ll}\text { A Fitting function parameters } & 13\end{array}$

\section{Introduction}

Numerous and different data collected during the last years have established the Standard Cosmological Model as a simple framework showing a remarkable agreement with observations. This model is based on Einstein's General Relativity and a homogeneous and isotropic ansatz for the metric. However, in addition to ordinary matter, two new elements need to be added: a cosmological constant or other form of dark energy to account for the late time acceleration of the universe, and dark matter (DM) to explain the formation and dynamics of cosmic structures. However, despite the multiple efforts, the fundamental nature of DM remains still as an open problem. There are strong astrophysical evidences for DM from galactic to cosmological scales, but the interactions with ordinary matter have not been probed beyond gravitational effects. In this sense, both direct and indirect DM searches are fundamental to explore particle models of DM.

In the framework of indirect searches, the observation of gamma-ray fluxes from astrophysical sources represents one of the main approaches to the DM puzzle. If DM annihilates into SM particles, the subsequent chains of decay and hadronization of unstable products produce gamma-ray photons generically. The observation of this signal is highly affected by astrophysical uncertainties in the gamma-ray backgrounds and in the DM densities and distribution. On the other hand, depending on both astrophysical and particle physics models of DM, the signature could be distinguishable from the background. Appealing targets for gamma-ray observations of annihilating DM are mainly selected according to the abundance of DM in the source and their distance. Galaxy clusters, dwarfs spheroidal galaxies (dSph) or the galactic center (GC) of the Milky Way are traditional targets of interest. Galaxy clusters are very rich in DM, but they are very distant objects. DSphs are also characterized by high DM densities but at much shorter distances. In any case, despite their proximity, observations of dSphs have not been able to detect any gamma-ray flux signature over the background so far $[1,2]$. On the other hand, the GC represents a very close target, but the complexity of the region, due to the high concentration of sources, makes the analysis quite involved.

In this work, we will focus on the analysis of very high energy (VHE) gamma-rays coming from the GC. Different observations from the GC have been reported by several 
collaborations such as CANGAROO [3], VERITAS [4], HESS [5, 6], MAGIC [7] and FermiLAT $[8,9]$. In particular, we will analyze the data collected by the HESS collaboration during the years 2004, 2005, and 2006 associated with the HESS J1745-290 GC source [6]. The interpretation of these fluxes as DM signal has been widely discussed in the literature from the very early days of the publication of the observed data [10-13]. It was concluded that the spectral features of these gamma-rays disfavored the DM origin $[13,14]$. However, in recent studies $[15,16]$, it has been pointed out that these fluxes are compatible with the spectral signal of DM annihilation provided it is complemented with a simple background. This extra source of gamma-rays can be originated by radiative processes generated by particle acceleration in the vicinity of Sgr A East supernova and the supermassive black hole [17].

In this work, we present a systematic study of this assumption: in section 2, we show an analysis of the source, while a brief review of the gamma-ray flux coming from annihilating DM in galactic sources is presented in section 3. The fit of the HESS data with a total fitting function given by the combination of the background power law component with annihilating DM signature is discussed in section 4. In section $\mathrm{V}$, we include in the analysis the data collected by Fermi-LAT from the same region. We summarize the main results of our work and prospects for future analyses in section 6. Finally, an appendix provides useful details for reproducing the statistical study performed in these analyses.

\section{HESS J1745-290 data}

DM is expected to be clumped in the central region of standard galaxies. In this sense, the central part of the Milky Way could be an important source of gamma-rays produced in the DM annihilation processes. Because of its closeness, the GC represents a very appealing target for the indirect search of DM, but the complex nature of this area makes the identification of the sources quite difficult. Several sources have been detected not only in the gamma-ray, but also in the infrared and X-ray ranges of the spectrum. The absence of variability in the collection of HESS J1745-290 data in the TeV scale, during the years 2004, 2005 and 2006, suggests that the emission mechanism and emission regions differ from those invoked in the variable infrared and X-ray emissions [18]. The significance of the signal reduces to few tenths of degree [6], but the angular distribution of the very high energy gamma-ray emission shows the presence of an adjunctive diffuse component. The fundamental nature of this source is still unclear. These gamma-rays could have been originated by particle propagation $[9,17]$ in the neighborhood of the Sgr A East supernova remnant and the supermassive black hole Sgr A, both located at the central region of our galaxy $[14,19]$. If it was originated by the DM distribution, the morphology of the source requires a very compressed DM structure. In fact, it has been claimed $[20,21]$ that baryonic gas could account for similar effects when falling to the central region, modifying the gravitational potential and increasing the DM density in the center (see however $[22,23]$ ). If this is the case, the DM annihilating fluxes are expected to be enhanced by an important factor with respect to DM alone simulations, such as the classical NFW profile [21].

In previous works [6, 19], important deviations from a power law spectrum have been already reported, and a cut-off at several tens of $\mathrm{TeVs}$ has been proved as a distinctive feature of the spectrum. For instance, the observed data were compared to a simple power law:

$$
\frac{d \Phi_{B g}}{d E}=\Phi_{0} \cdot\left(\frac{E}{\mathrm{GeV}}\right)^{-\Gamma}
$$




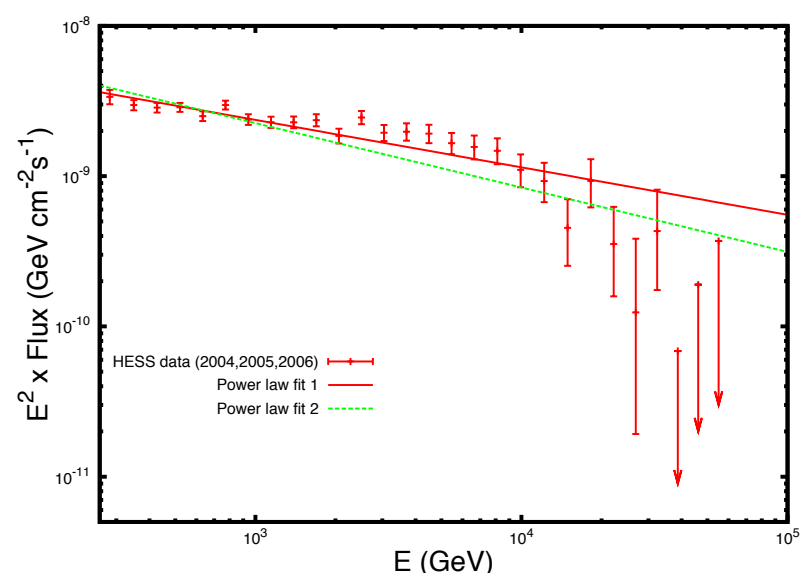

\begin{tabular}{|ccc|}
\hline & Power law 1 & Power law 2 \\
\hline$\Phi_{0}$ & $20.9 \pm 5.1$ & $43.5 \pm 14.8$ \\
$\Gamma$ & $2.32 \pm 0.03$ & $2.43 \pm 0.05$ \\
$\chi^{2} /$ dof & $2.48(57 / 23)$ & $5.30(137 / 26)$ \\
\hline
\end{tabular}

Figure 1. Collection of the HESS data (2004,2005 and 2006) fitted with two simple power law background. The full line (Power law 1) shows the fit with 23 dof, without take into account the upper limit constraints on the flux given by the last three points. They are included in the Power law 2 fit with 26 dof (dotted line). The parameters of the fit can be found in the table. Because of the poorness of both the fits $\left(\chi^{2} /\right.$ dof $=2.48$ with $\Gamma=2.32 \pm 0.03$ for Power law 1 , and $\chi^{2} / d o f=5.30$ with $\Gamma=2.43 \pm 0.03$ for Power law 2), they represent just an upper limit of the flux. These results are consistent with previous studies $[6,19]$.

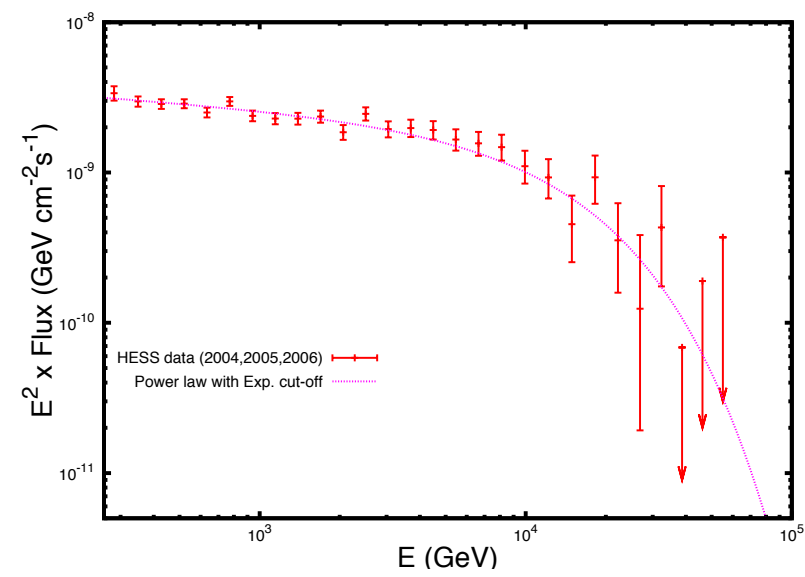

\begin{tabular}{|cc|}
\hline & Exp Cut-off law \\
\hline$\Phi_{0}$ & $6.24 \pm 1.53$ \\
$\Gamma$ & $2.12 \pm 0.04$ \\
$E_{\text {cut }}$ & $13.8 \pm 2.3$ \\
$\chi^{2} /$ dof & $0.87(21 / 25)$ \\
\hline
\end{tabular}

Figure 2. The same as in figure 1, but the power law is modified with an exponential energy cut-off. The signal is well fitted in this case with the results given in the attached table.

and a power law with high energy exponential cut-off:

$$
\frac{d \Phi_{B g}}{d E}=\Phi_{0} \cdot\left(\frac{E}{\mathrm{GeV}}\right)^{-\Gamma} \cdot e^{-\frac{E}{E_{\mathrm{cut}}}}
$$

where $\Phi_{0}$ is the flux normalization, $\Gamma$ is the spectral index and $E_{\text {cut }}$ is the cut-off energy. The measured spectrum for the three-year dataset ranges from $260 \mathrm{GeV}$ to $60 \mathrm{TeV}$. We have reproduced these analyses with the results that can be found in figures 1 and 2. They are consistent with previous works $[6,19]$, since the spectrum is well described by a power law with exponential cut-off (figure 2), while a simple power law is clearly inconsistent (figure 1). 


\section{Gamma-rays from dark matter annihilation}

If the signal observed from the GC is a combination of gamma-ray photons from annihilating DM and a background, the total fitting function for the observed differential gamma-ray flux will be:

$$
\frac{d \Phi_{\mathrm{Tot}}}{d E}=\frac{d \Phi_{B g}}{d E}+\frac{\Phi_{D M}}{d E} .
$$

We will assume a simple power law background parameterized as:

$$
\frac{d \Phi_{B g}}{d E}=B^{2} \cdot\left(\frac{E}{\mathrm{GeV}}\right)^{-\Gamma} .
$$

On the other hand, the differential gamma-ray flux originated from DM annihilation in galactic structures and substructures can be written generically as:

$$
\frac{d \Phi_{\mathrm{DM}}}{d E}=\sum_{i}^{\text {channels }} \frac{\left\langle\sigma_{i} v\right\rangle}{2} \cdot \frac{d N_{i}}{d E} \cdot \frac{\Delta \Omega\langle J\rangle_{\Delta \Omega}}{4 \pi M},
$$

where $\left\langle\sigma_{i} v\right\rangle$ are the thermal averaged annihilation cross-sections of two DM particles into SM particles (also labeled by the subindex i). We are assuming that the DM particle is its own antiparticle. $M$ is the mass of the DM particle, and the number of photons produced in each annihilating channel $d N_{i} / d E$, involves decays and/or hadronization of unstable products such as quarks and leptons. Because of the non-perturbative QCD effects, the calculation of $d N_{i} / d E$ requires Monte Carlo events generators such as PYTHIA [24]. In our analysis, we will focus on gamma-rays coming from external bremsstrahlung and fragmentation of SM particle-antiparticle pairs produced by DM annihilation. We will ignore DM decays, the possible production of monoenergetic photons, N-body annihilations (with $N>2$ ), or photons produced from internal bremsstrahlung, that are model dependent. In particular and in order to simplify the discussion and provide useful information for a general analysis, we will consider DM annihilation into each single channel of SM particle-antiparticle pairs, i.e.

$$
\frac{d \Phi_{\mathrm{DM}}^{i}}{d E}=A_{i}^{2} \cdot \frac{d N_{i}}{d E},
$$

where

$$
A_{i}^{2}=\frac{\left\langle\sigma_{i} v\right\rangle \Delta \Omega\langle J\rangle_{\Delta \Omega}}{8 \pi M^{2}}
$$

is a new constant to be fitted together with the DM particle mass $M$, and the background parameters $B$ and $\Gamma$. The astrophysical factor $\langle J\rangle_{\Delta \Omega}$ of eq. (3.3) will be also indirectly fitted by means of the parameter $A_{i}$. In its most general expression, it is computed in the direction $\Psi$ defined by the line of observation towards the GC:

$$
\langle J\rangle_{\Delta \Omega}=\frac{1}{\Delta \Omega} \int_{\Delta \Omega} \mathrm{d} \Omega \int_{0}^{l_{\max }(\Psi)} \rho^{2}[r(l)] d l(\Psi),
$$

where $l$ is the distance from the Sun to any point in the halo. The radial distance $r$ is measured from the GC, and is related to $l$ by $r^{2}=l^{2}+D_{\odot}^{2}-2 D_{\odot} l \cos \Psi$, where $D_{\odot} \simeq 8.5$ $\mathrm{kpc}$ is the distance from the Sun to the center of the Galaxy. The distance from the Sun to the edge of the halo in the direction $\theta$ is $l_{\max }=D_{\odot} \cos \theta+\sqrt{r^{2}-D_{\odot}^{2} \sin \theta}$. 
The photon flux must be averaged over the solid angle of the detector, that is typically of order $\Delta \Omega=2 \pi(1-\cos \Psi) \simeq 10^{-5}$ for detectors with sensitivities in the TeV energy scale, such as the HESS Cherenkov telescopes array. The dark halo in the GC is usually modeled by the NFW profile [25]:

$$
\rho(r) \equiv \frac{N}{r\left(r-r_{s}\right)^{2}},
$$

where $N$ is the overall normalization and $r_{s}$ the scale radius. This profile is in good agreement with non-baryonic cold DM simulations of the GC. In this case and accounting for just annihilating DM, the astrophysical factor is: $\left\langle J_{(2)}^{\mathrm{NFW}}\right\rangle \simeq 280 \cdot 10^{23} \mathrm{GeV}^{2} \mathrm{~cm}^{-5}$, value that we will use as standard reference.

\section{Single-channel fits}

As commented before, the particle model part of the differential gamma-ray flux expected from the GC is simulated by means of Monte Carlo event generators, such as PYTHIA [24]. However, the fact that simulations have to be performed for fixed DM mass implies that we cannot obtain explicit $M$ dependence for the photon spectra. In order to overcome this limitation, the simulated spectra of each annihilation channel has been reproduced with the analytic fitting functions provided in ref. [26] in terms of the WIMP mass, by means of mass dependent parameters. The combination of such simulated spectra with a power law background (eq. (3.1)) is finally fitted. We assume a typical experimental resolution of $15 \%$ $(\Delta E / E \simeq 0.15)$ and a perfect detector efficiency.

For quarks (except the top) electron and $\tau$ leptons, the most general formula needed to reproduce the behaviour of the differential number of photons in an energy range may be written as:

$$
\begin{aligned}
\frac{d N}{d E}= & {\left[a_{1} \exp \left(-b_{1}\left(\frac{E}{M}\right)^{n_{1}}-b_{2}\left(\frac{E}{M}\right)^{n_{2}}-\frac{c_{1}}{\left(\frac{E}{M}\right)^{d_{1}}}+\frac{c_{2}}{\left(\frac{E}{M}\right)^{d_{2}}}\right)\right.} \\
& \left.+q\left(\frac{E}{M}\right)^{1.5} \ln \left[p\left(1-\left(\frac{E}{M}\right)\right)\right] \frac{\left(\frac{E}{M}\right)^{2}-2\left(\frac{E}{M}\right)+2}{\left(\frac{E}{M}\right)}\right] E^{-1.5} M^{0.5} .
\end{aligned}
$$

The value of the different parameters change with the SM particle annihilation channel and in some cases, with the range of the WIMP mass. The cases of interest are described below and the value of the parameters reported in appendix A. In the case of the electron-positron channel, the only contribution to the gamma-rays flux comes from the bremsstrahlung of the final particles. Therefore, in the previous expression (4.1) the exponential contribution is absent, $q=\alpha_{\mathrm{QED}} / \pi$, and $p=\left(M / m_{e^{-}}\right)^{2}$. This choice of the parameters corresponds to the well-known Weizsäcker-Williams approximation (figure 3).

In the case of the $\mu^{+} \mu^{-}$channel, the exponential contribution in the expression above (4.1) is also absent. A proper fitting function for such a channel can be written as:

$$
\frac{\mathrm{d} N}{\mathrm{~d} E}=q\left(\frac{E}{M}\right)^{1.5} \ln \left[p\left(1-\left(\frac{E}{M}\right)^{l}\right)\right] \frac{\left(\frac{E}{M}\right)^{2}-2\left(\frac{E}{M}\right)+2}{\left(\frac{E}{M}\right)} E^{-1.5} M^{0.5} .
$$

All the parameters are here mass dependent and their expression for a range of mass $10^{3} \mathrm{GeV} \lesssim M \lesssim 5 \times 10^{4} \mathrm{GeV}$ are reported in table 1 . The best fit for the $\mu$ lepton is shown in figure 4 . 
The $\tau^{+} \tau^{-}$spectrum needs the entire eq. (4.1) for an accurate analysis. The value of the mass independent parameter and the expression of the mass dependent ones used in this work are reported in table 2. The best fit for the $\tau$ lepton is shown in figure 5 .

The value of the mass independent parameter and the expression of the mass dependent ones in (4.1) for the $u \bar{u}$ channel are reported in table 3. The analytic fitting function is given for mass values up $8000 \mathrm{GeV}$, because of limitations in the Monte Carlo event generator PYTHIA software. An extrapolation up to larger values of the mass has been performed. In this case the best fit is shown in figure 6 . The parameters for the $d \bar{d}$ channel are given in table 4. This channel provides the best fit (See figure 7) of all those considered in the paper and is used as a reference for comparison with other channels. The parameters for the $s \bar{s}, c \bar{c}$ and $b \bar{b}$ are reported in tables 5,6 and 7. The best fit of these hadronic channels are shown in figures 8, 9 and 10 .

The $t \bar{t}$ needs a different fitting function:

$$
\frac{d N}{d E}=a_{1} \exp \left(-b_{1}\left(\frac{E}{M}\right)^{n_{1}}-\frac{c_{1}}{\left(\frac{E}{M}\right)^{d_{1}}}-\frac{c_{2}}{\left(\frac{E}{M}\right)^{d_{2}}}\right)\left\{\frac{\ln \left[p\left(1-\left(\frac{E}{M}\right)^{l}\right)\right]}{\ln p}\right\}^{q} E^{-1.5} M^{0.5} .
$$

The value of the mass independent parameter and the expression of the mass dependent ones (in the selected range of mass) are reported in table 8 . The best fit for the $t \bar{t}$ channel by using eq. (4.3) is shown in figure 11.

For the $W$ and $Z$ gauge bosons the parametrization is:

$$
\frac{d N}{d E}=a_{1} \exp \left(-b_{1}\left(\frac{E}{M}\right)^{n_{1}}-\frac{c_{1}}{\left(\frac{E}{M}\right)^{d_{1}}}\right)\left\{\frac{\ln \left[p\left(j-\frac{E}{M}\right)\right]}{\ln p}\right\}^{q} E^{-1.5} M^{0.5},
$$

where the value of the parameters used in this study are reported in table 9 . The best fits for the $W^{+} W^{-}$and $Z Z$ channels are shown in figures 12 and 13 .

The best fit is provided by the $d \bar{d}$ channel with $\chi^{2} / d o f=0.73$ for a total of 24 degrees of freedom (dof). In any case, other hadronic channels such as $u \bar{u}$ (see figure 6) or $s \bar{s}$, also provide very good fits within $1 \sigma$. In the same way, softer spectra as the one provided by $t \bar{t}$ (see figure 11), $W^{+} W^{-}$(figure 12) or $Z Z$ (figure 13) channels are consistent with data without statistical significance difference. On the contrary, leptonic channels (not only $e^{+} e^{-}$, or $\mu^{+} \mu^{-}$but also $\tau^{+} \tau^{-}$, figures 3,4 and 5), $c \bar{c}$ and $b \bar{b}$ channels (figure 9 and 10) are ruled out with more than $99 \%$ confidence level when compared to the best channel. It is interesting to note that taking into account all the channels that provide a good fit, the DM mass is constrained to $15 \mathrm{TeV} \lesssim M \lesssim 110 \mathrm{TeV}$ within $2 \sigma$. The lighter values are consistent with hadronic annihilations $(u \bar{u})$ and the heavier ones with the annihilation in $t \bar{t}$, that is more similar to electroweak channels.

\section{FERMI 1FGL J1745.6-2900 data}

It has been argued that the Fermi-LAT source 1FGL J1745.6-2900 and the previously considered HESS source J1745-290 are spatially coincident [27]. In [9] data from the first 25 months of observations of the mentioned Fermi-LAT source have been analyzed. It has been shown that the observed spectrum in the range $100 \mathrm{MeV}-300 \mathrm{GeV}$ can be very well described by a broken power law with a break energy of $E_{b r}=2.0_{-1.0}^{+0.8} \mathrm{GeV}$, and slopes $\Gamma_{1}=2.20 \pm 0.04$ and $\Gamma_{2}=2.68 \pm 0.05$ for lower and higher energies than $E_{b r}$ respectively. Notice that the fitted value of $\Gamma_{2}$ from Fermi-LAT data is in very good agreement with the spectral index 


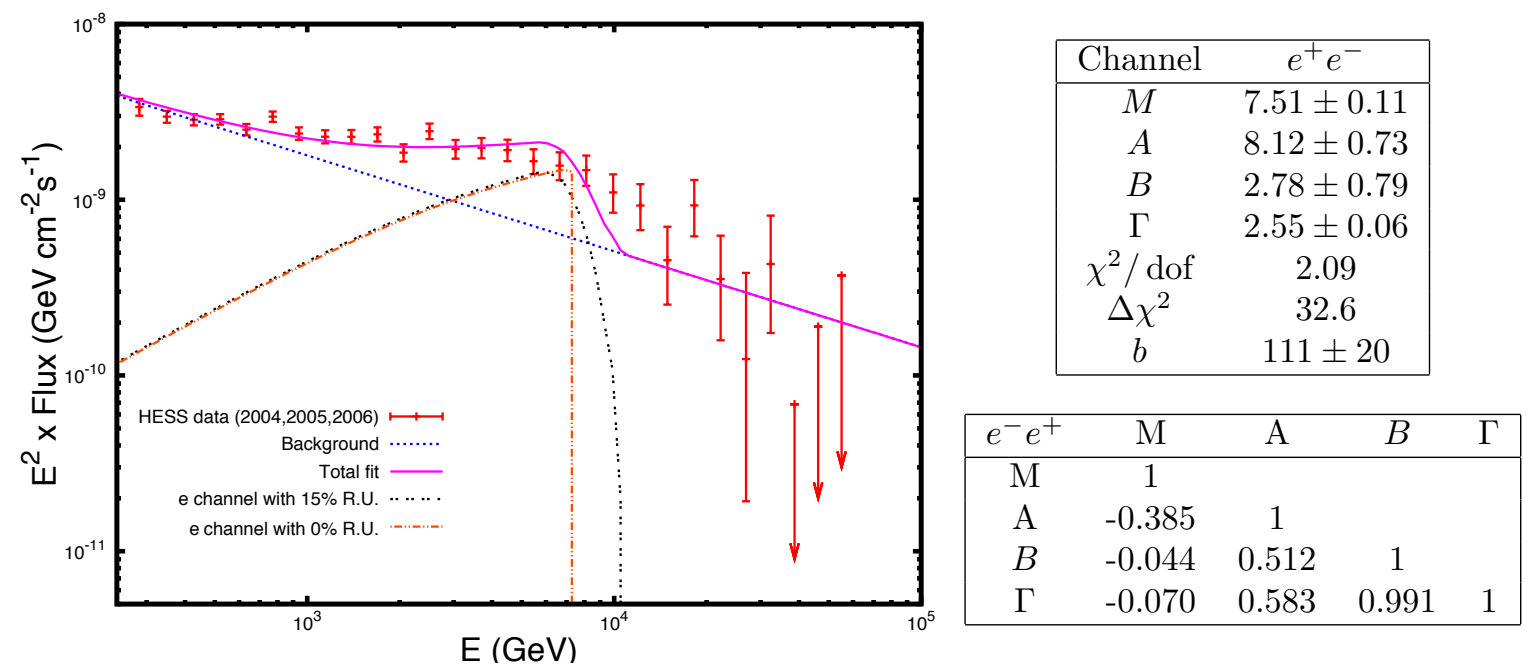

Figure 3. $M(\mathrm{TeV}), A\left(10^{-7} \mathrm{~cm}^{-1} \mathrm{~s}^{-1 / 2}\right), B\left(10^{-4} \mathrm{GeV}^{-1 / 2} \mathrm{~cm}^{-1} \mathrm{~s}\right)$. Best fit to the HESS J1745-290 collection of data (years 2004, 2005, and 2006 [6]) in the case that the DM contribution came entirely from annihilation into $e^{+} e^{-}$. The full line shows the total fitting function. The poor quality of the fit is evident $\left(\chi^{2} / d o f=2.09\right)$. The dotted line shows the simple power law background component with spectral index $\Gamma=2.55 \pm 0.06$, while the DM component with a $15 \%$ of resolution uncertainity (R. U.) is given by the double-dotted line with a normalization parameter $A$. The dotted-dashed line shows the contribution of annihilating DM into electronpositron pairs without taking into account such a resolution. On the top right table, we present a summary of the best fitting parameters. $\Delta \chi^{2}$ correponds to the difference with respect to the best fit channel $(d \bar{d})$. On the bottom right table, we show the correlation matrix elements of this statistical analysis.

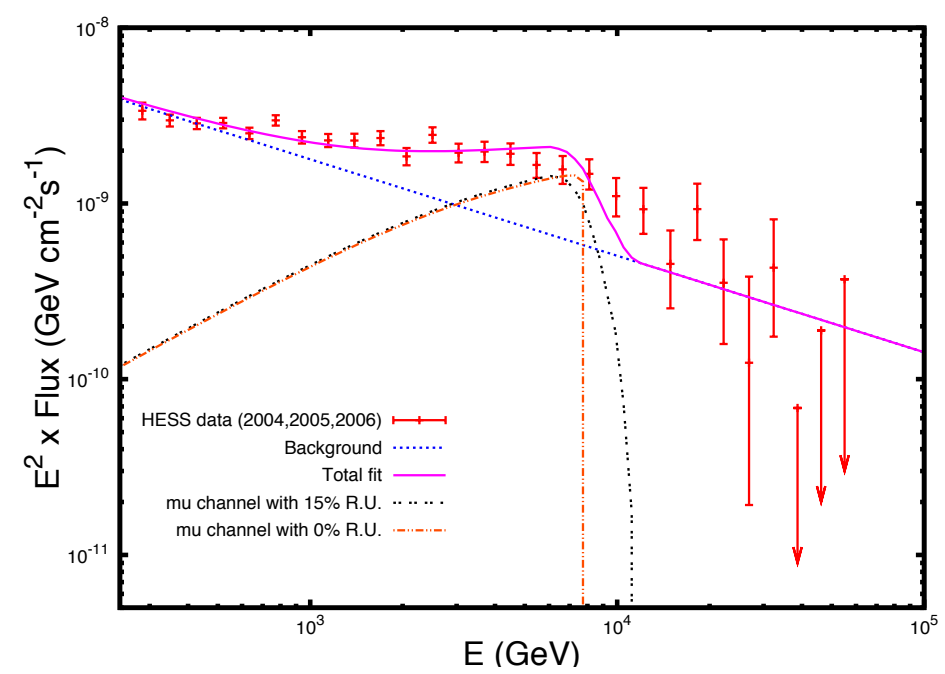

\begin{tabular}{|cc|}
\hline Channel & $\mu^{+} \mu^{-}$ \\
\hline$M$ & $7.89 \pm 0.21$ \\
$A$ & $21.2 \pm 1.92$ \\
$B$ & $2.81 \pm 0.53$ \\
$\Gamma$ & $2.55 \pm 0.06$ \\
$\chi^{2} /$ dof & 2.04 \\
$\Delta \chi^{2}$ & 31.4 \\
$b$ & $837 \pm 158$ \\
\hline
\end{tabular}

\begin{tabular}{|ccccc|}
\hline$\mu^{+} \mu^{-}$ & $\mathrm{M}$ & $\mathrm{A}$ & $B$ & $\Gamma$ \\
\hline $\mathrm{M}$ & 1 & & & \\
$\mathrm{~A}$ & -0.431 & 1 & & \\
$B$ & -0.052 & 0.515 & 1 & \\
$\Gamma$ & -0.081 & 0.584 & 0.991 & 1 \\
\hline
\end{tabular}

Figure 4. The same as figure 3 in the case of the $\mu^{+} \mu^{-}$annihilation channel. The fit is only slightly better than for the $e^{+} e^{-}$case.

of the diffuse background obtained from HESS data in our previous analyses. Indeed, at the $95 \%$ confidence level, the allowed range for the spectral index of the diffuse background is $2.6 \lesssim \Gamma \lesssim 3.7$. In this case, the lower values are consistent with all the allowed channels, but the higher values are only accessible to the light quark channels. In any case all the channels that provide a good fit to HESS data are also consistent with the spectral index observed by Fermi-LAT. 


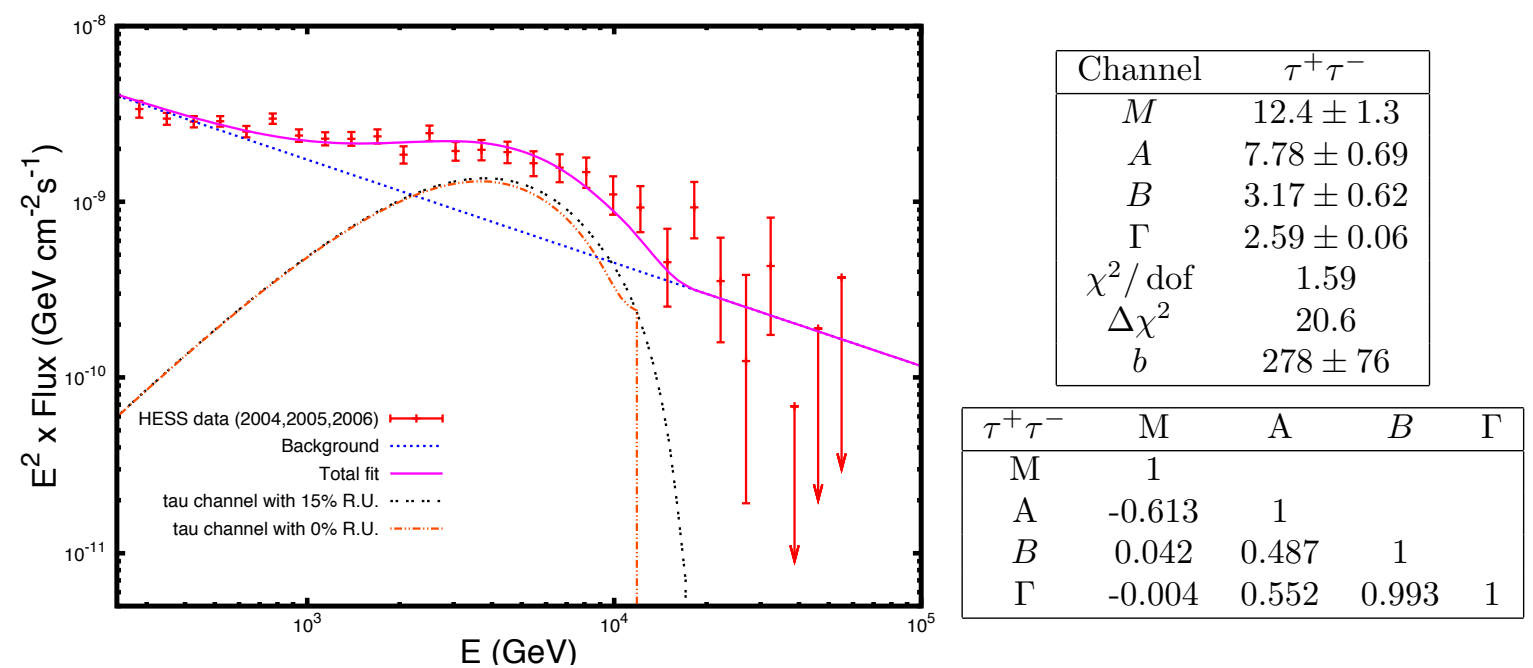

Figure 5. The same as figure 3 in the case of the $\tau^{+} \tau^{-}$annihilation channel. This channel provides the best leptonic fit, although it is not enough to be consistent with data.

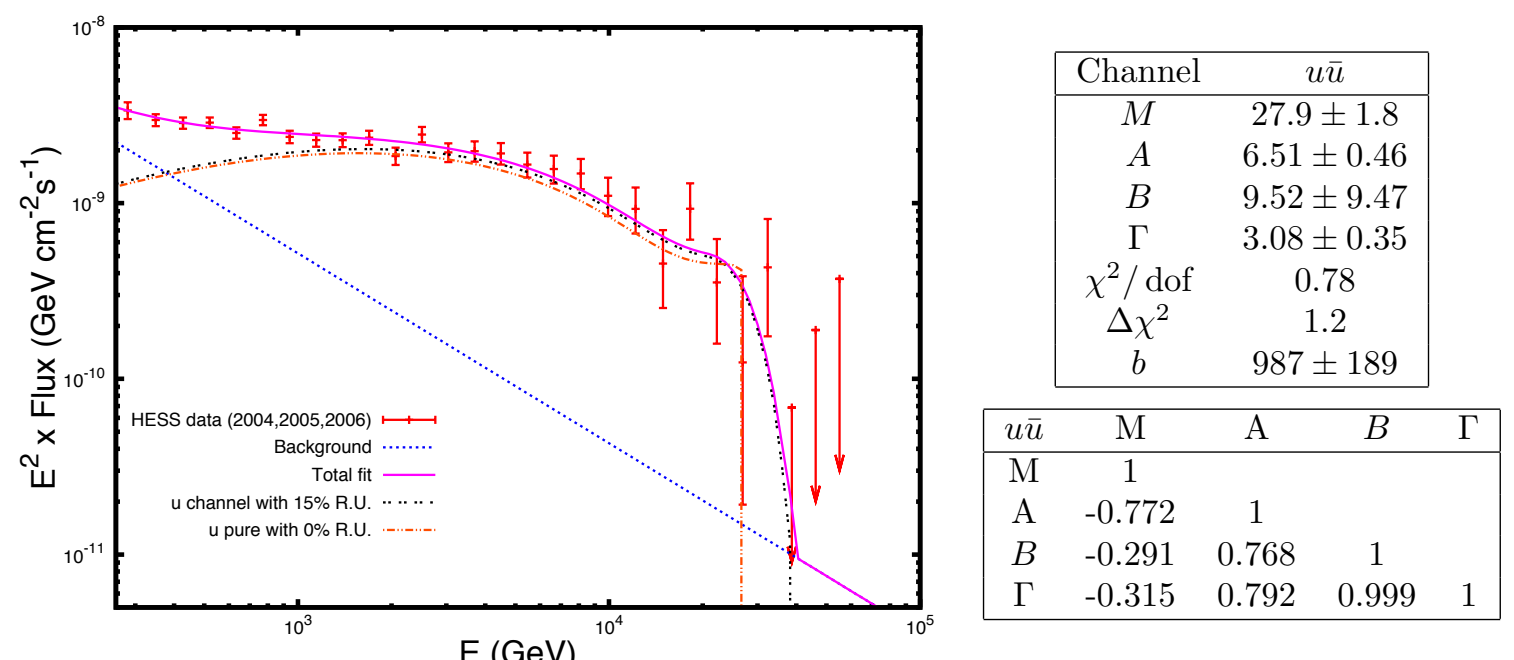

Figure 6. The same as figure 3 in the case of the $u \bar{u}$ annihilation channel. The cut-off in the energy spectrum of this hadronic channel reproduces the cut-off in the data, giving good consistency.

In figure 14 , we show the case of the $W^{+} W^{-}$channel to illustrate this consistency. Both the signal and background parameters are compatible with the $W^{+} W^{-}$channel fit without the Fermi-LAT data. With the new data, the analysis even improves to $\chi^{2} / d o f=0.75$. This interpretation implies that the Fermi-LAT telescope is able to detect just the background component of the total energy spectrum of the gamma-ray emission associated with DM.

With the fit of the parameter $A$ (eq. (3.5)) and by assuming a standard thermal cross section of $\langle\sigma v\rangle=3 \cdot 10^{-26} \mathrm{~cm}^{3} \mathrm{~s}^{-1}$, we can get an estimation of the astrophysical factor $\langle J\rangle$. We find $10^{25} \mathrm{GeV}^{2} \mathrm{~cm}^{-5} \lesssim\left\langle J^{\mathrm{NFW}}\right\rangle \lesssim 10^{26} \mathrm{GeV}^{2} \mathrm{~cm}^{-5}$. It implies that the boost factors $b \equiv\langle J\rangle /\left\langle J^{\mathrm{NFW}}\right\rangle$ spread on a range between two and three orders of magnitude. It is interesting to note that the enhancement of the distribution of DM required to fit the data is compatible with the expectation of N-body simulations when the effect of the baryonic matter in the inner part of GC are taken into account [21]. 


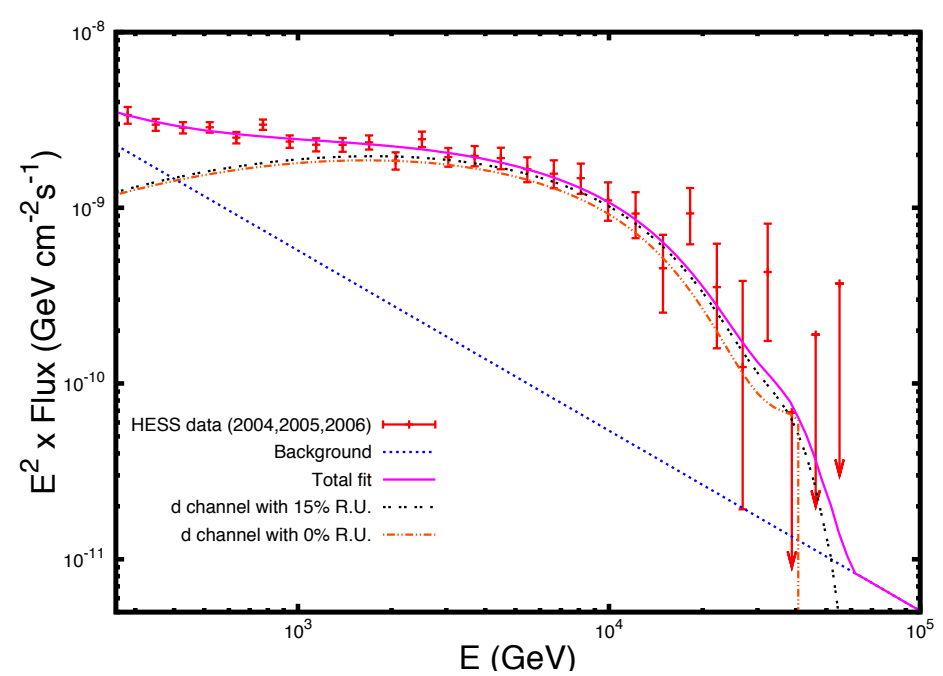

\begin{tabular}{|c|c|c|}
\hline Channel & \multicolumn{2}{|c|}{$d \bar{d}$} \\
\hline$M$ & \multicolumn{2}{|c|}{$42.0 \pm 4.4$} \\
\hline$A$ & \multicolumn{2}{|c|}{$4.88 \pm 0.48$} \\
\hline$B$ & \multicolumn{2}{|c|}{$8.26 \pm 7.86$} \\
\hline$\Gamma$ & \multicolumn{2}{|c|}{$3.03 \pm 0.34$} \\
\hline$\chi^{2} / \operatorname{dof}$ & \multicolumn{2}{|c|}{0.73} \\
\hline$\Delta \chi^{2}$ & \multicolumn{2}{|c|}{0.0} \\
\hline$b$ & \multicolumn{2}{|c|}{$1257 \pm 361$} \\
\hline $\mathrm{M}$ & $\mathrm{A}$ & $B$ \\
\hline 1 & & \\
\hline-0.883 & 1 & \\
\hline-0.435 & 0.764 & 1 \\
\hline-0.468 & 0.793 & 0.998 \\
\hline
\end{tabular}

Figure 7. The same as figure 3 in the case of the $d \bar{d}$ annihilation channel. The $\chi^{2} / d o f$ value associated with this analysis is the lowest one obtained in this work and it has been taken as reference to compute $\Delta \chi^{2}$.

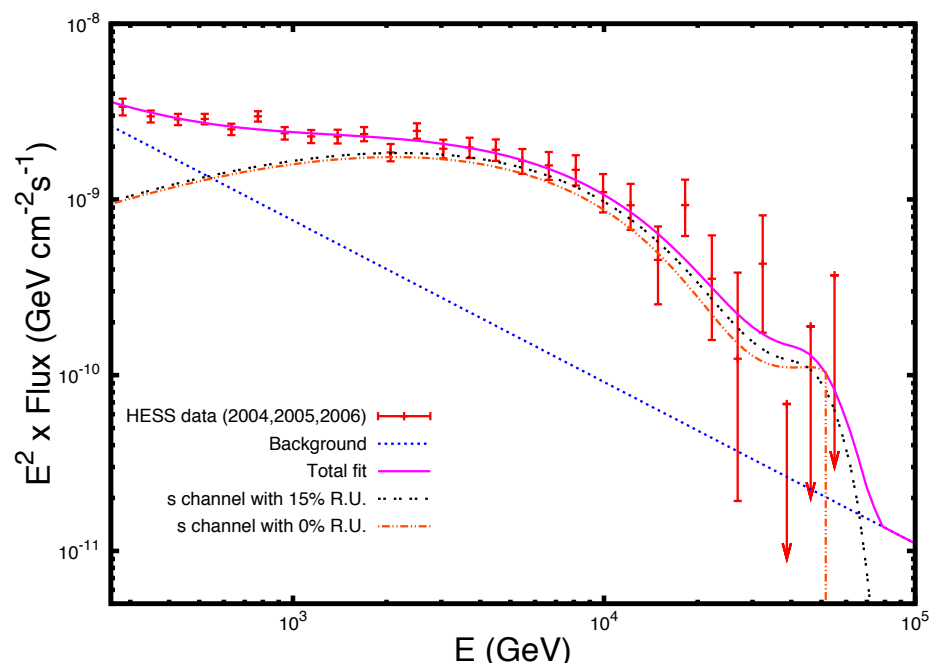

\begin{tabular}{|c|c|c|}
\hline Channel & \multicolumn{2}{|c|}{$s \bar{s}$} \\
\hline$M$ & \multicolumn{2}{|c|}{$53.9 \pm 6.2$} \\
\hline$A$ & \multicolumn{2}{|c|}{$4.85 \pm 0.57$} \\
\hline$B$ & \multicolumn{2}{|c|}{$6.59 \pm 5.43$} \\
\hline$\Gamma$ & \multicolumn{2}{|c|}{$2.92 \pm 0.29$} \\
\hline$\chi^{2} / \operatorname{dof}$ & \multicolumn{2}{|c|}{0.90} \\
\hline$\Delta \chi^{2}$ & \multicolumn{2}{|c|}{4.1} \\
\hline$b$ & \multicolumn{2}{|c|}{$2045 \pm 672$} \\
\hline $\mathrm{M}$ & $\mathrm{A}$ & $B$ \\
\hline 1 & & \\
\hline-0.852 & 1 & \\
\hline-0.410 & 0.784 & 1 \\
\hline-0.444 & 0.812 & 0.998 \\
\hline
\end{tabular}

Figure 8. The same as figure 3 in the case of the $s \bar{s}$ annihilation channel.

\section{Conclusions}

In this work, we have analyzed the possibility of explaining the gamma-ray data observed by HESS from the central part of our galaxy by being partially produced by DM annihilation. The complexity of the region and the ambiguous localisation of the numerous emitting sources inside this region, seems to validate the hypothesis of a background component. We have proved that even DM annihilations into single channels of the SM provide good fits provided that the DM signal is complemented with a diffuse background compatible with Fermi LAT observations. The fits returns a DM mass between $15 \mathrm{TeV} \lesssim M \lesssim 110 \mathrm{TeV}$ and a background spectral index between $2.6 \lesssim \Gamma \lesssim 3.7$ within $2 \sigma$. Some channels are clearly preferred with respect to others, such as the $d \bar{d}$ for the quark channels with $\chi^{2} / d o f=0.73$, or the $W^{+} W^{-}$ and $Z Z$ channels with $\chi^{2} / d o f=0.84$ and $\chi^{2} / d o f=0.85$. On the contrary, the leptonic channels are seriously disfavored. However, for a particular DM candidate, several channels 

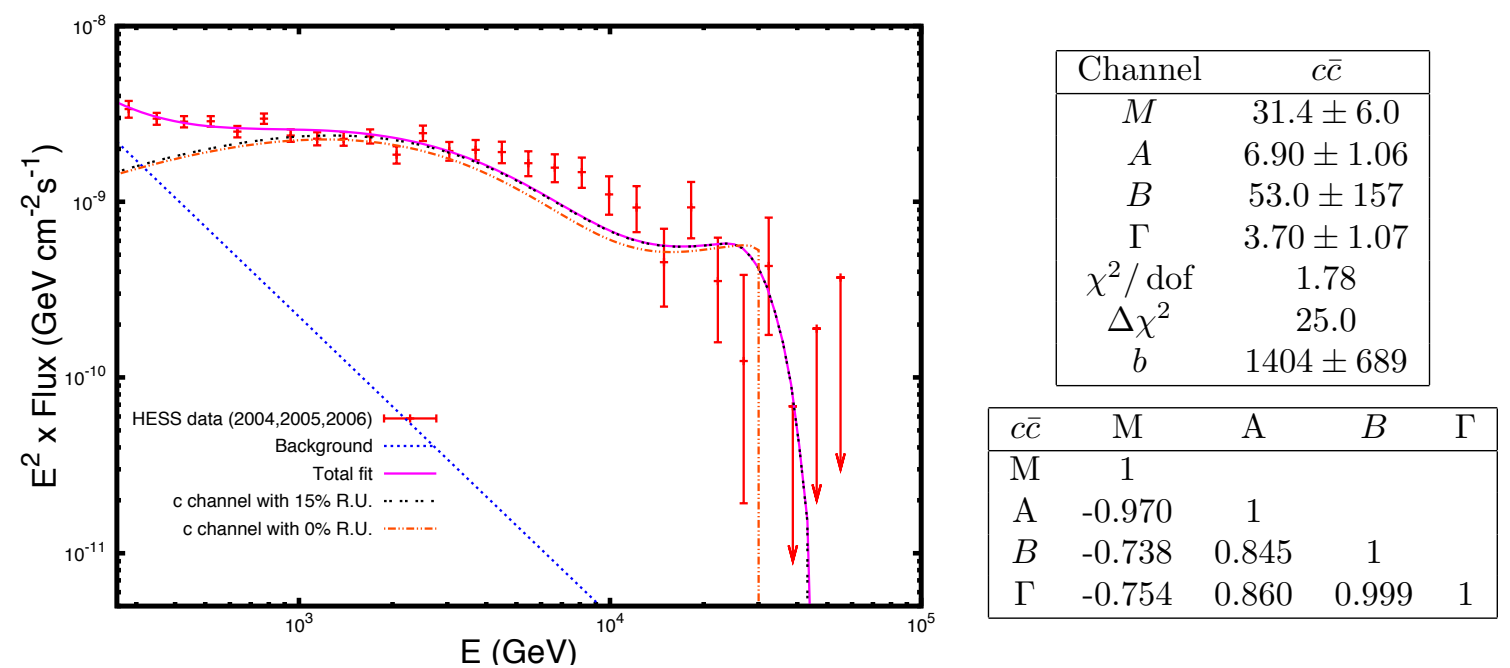

Figure 9. The same as figure 3 but for the $c \bar{c}$ channel. This is the quark channel most disfavored by the HESS data.

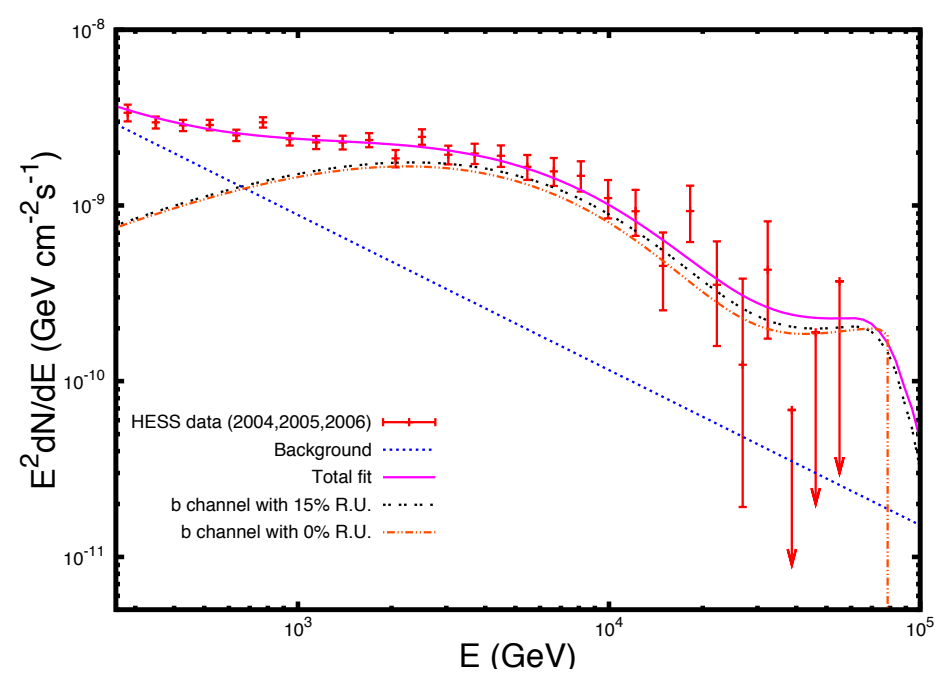

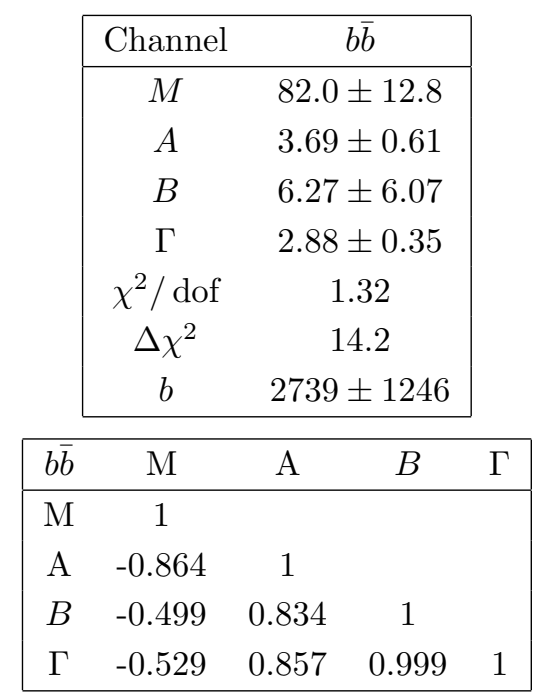

Figure 10. The same as figure 3 for the $b \bar{b}$ annihilation channel. Together with the $c \bar{c}$ channel, is the only hadronic channel disfavored by the HESS data.)

can actually contribute. In this situation, the single channel analysis presented in this work can be helpful as a guide but it is necessary to know that a combination of channels can work in a qualitatively different way. In any case, it is important to take into account that in an important amount of models, only one channel dominates the annihilation and the single channel approximation works with high accuracy.

The morphology of the signal is consistent with compressed dark halos by taking into account baryonic dissipation with boost factors of $b \sim 10^{3}$ for typical thermal cross sections. We would like to comment that the boost factors values presented in the tables are computed under the assumption of standard thermal annihilating dark matter. In a general approach, i.e. for decaying dark matter or for dark matter candidates, whose abundances are not reached by thermal freeze-out decoupling, the boost factors do not apply. However, thermal annihilating dark matter is a well motivated framework, and it is interesting to in- 


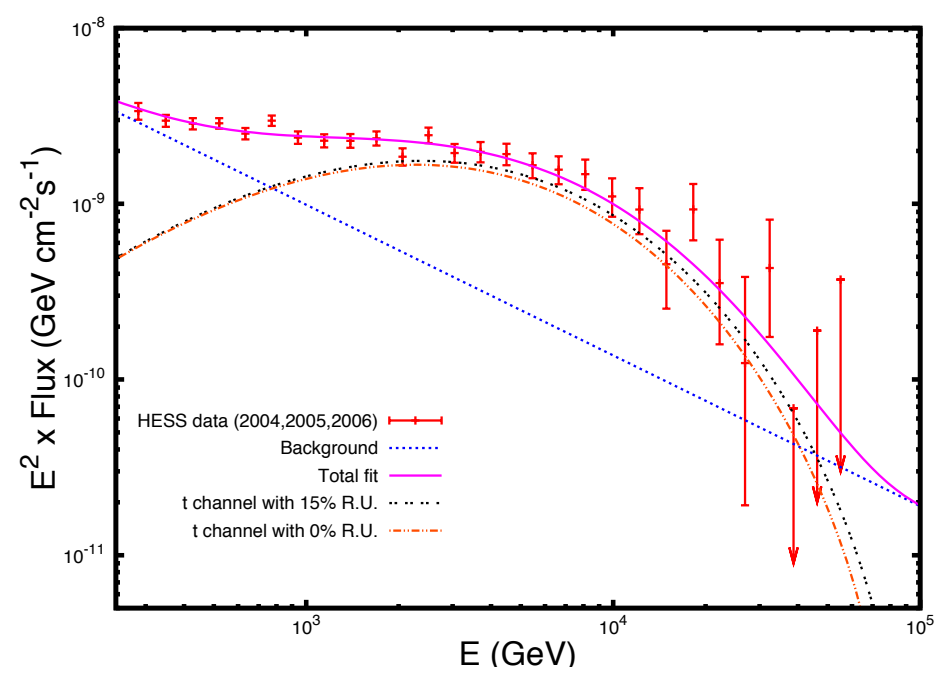

\begin{tabular}{|cc|}
\hline Channel & $t \bar{t}$ \\
\hline$M$ & $87.7 \pm 8.2$ \\
$A$ & $3.68 \pm 0.34$ \\
$B$ & $6.07 \pm 3.34$ \\
$\Gamma$ & $2.86 \pm 0.19$ \\
$\chi^{2} /$ dof & 0.88 \\
$\Delta \chi^{2}$ & 3.6 \\
$b$ & $3116 \pm 820$ \\
\hline
\end{tabular}

\begin{tabular}{|ccccc|}
\hline$t \bar{t}$ & $\mathrm{M}$ & $\mathrm{A}$ & $B$ & $\Gamma$ \\
\hline $\mathrm{M}$ & 1 & & & \\
$\mathrm{~A}$ & -0.748 & 1 & & \\
$B$ & -0.175 & 0.721 & 1 & \\
$\Gamma$ & -0.216 & 0.756 & 0.998 & 1 \\
\hline
\end{tabular}

Figure 11. The same as figure 3 in the case of the $t \bar{t}$ annihilation channel. The qualitative features of this channel are more similar to the electroweak annihilations (see following figures) than to the hadronic ones (as it is shown in the previous figures). The quality of the fit is also good.

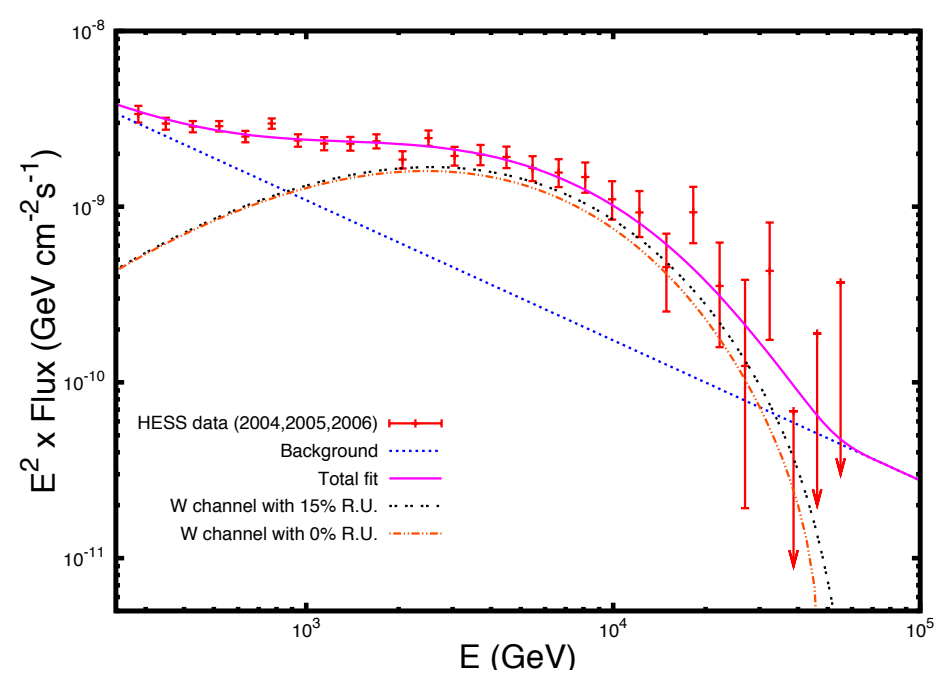

\begin{tabular}{|cc|}
\hline Channel & $W^{+} W^{-}$ \\
\hline$M$ & $48.8 \pm 4.3$ \\
$A$ & $4.98 \pm 0.40$ \\
$B$ & $5.18 \pm 2.23$ \\
$\Gamma$ & $2.80 \pm 0.15$ \\
$\chi^{2} /$ dof & 0.84 \\
$\Delta \chi^{2}$ & 2.6 \\
$b$ & $1767 \pm 419$ \\
\hline
\end{tabular}

\begin{tabular}{|ccccc|}
\hline$W^{+} W^{-}$ & $\mathrm{M}$ & $\mathrm{A}$ & $\mathrm{B}$ & $\Gamma$ \\
\hline $\mathrm{M}$ & 1 & & & \\
$\mathrm{~A}$ & -0.687 & 1 & & \\
$\mathrm{~B}$ & -0.038 & 0.681 & 1 & \\
$\Gamma$ & 0.008 & 0.636 & 0.997 & 1 \\
\hline
\end{tabular}

Figure 12. The same as figure 3 for the $W^{+} W^{-}$annihilation channel.

terpret the amount of gamma-rays observed by HESS in terms of such a hypothesis. We have adopted this approach in order to present our results. In this sense, it is important to note that the observed source of gamma-rays is very localized. It implies that if they are produced by annihilation or decay of dark matter, it has to be related to a very dense substructure or to a compressed halo. The latter case has been obtained in different simulations that take into account baryonic effects. Indeed it has been claimed [20, 21] that when baryonic gas is taken into account, it falls to the central region, modifying the gravitational potential. This fact increases the central dark matter density and produces typical enhancements of $b \sim 10^{3}$ in gamma ray fluxes [21].

On the other hand, the DM particle that may have originated these data needs to be heavier than $\sim 10 \mathrm{TeV}$. These large DM masses required for fitting the HESS data are not in 


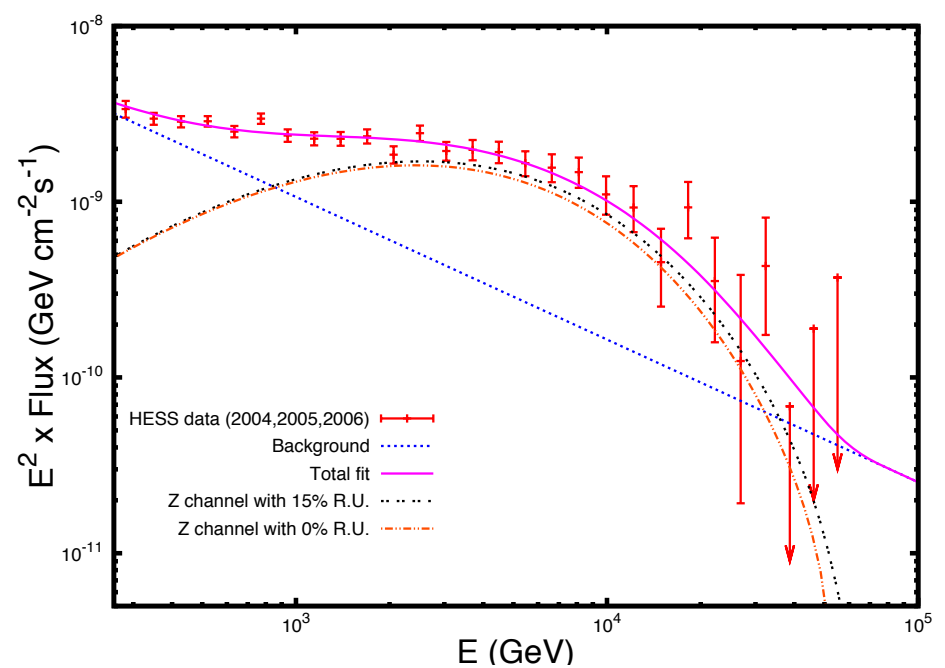

\begin{tabular}{|cc|}
\hline Channel & $Z Z$ \\
\hline$M$ & $54.5 \pm 4.9$ \\
$A$ & $4.73 \pm 0.40$ \\
$B$ & $5.38 \pm 2.45$ \\
$\Gamma$ & $2.81 \pm 0.16$ \\
$\chi^{2} /$ dof & 0.85 \\
$\Delta \chi^{2}$ & 2.9 \\
$b$ & $1988 \pm 491$ \\
\hline
\end{tabular}

\begin{tabular}{|ccccc|}
\hline$Z Z$ & $\mathrm{M}$ & $\mathrm{A}$ & $\mathrm{B}$ & $\Gamma$ \\
\hline $\mathrm{M}$ & 1 & & & \\
$\mathrm{~A}$ & -0.704 & 1 & & \\
$\mathrm{~B}$ & -0.042 & 0.660 & 1 & \\
$\Gamma$ & -0.088 & 0.703 & 0.997 & 1 \\
\hline
\end{tabular}

Figure 13. The same as figure 3 in the case of the $Z Z$ annihilation. The results for the $Z Z$ channel are very similar to the $W^{+} W^{-}$one. Both electroweak channels are consistent with the data.

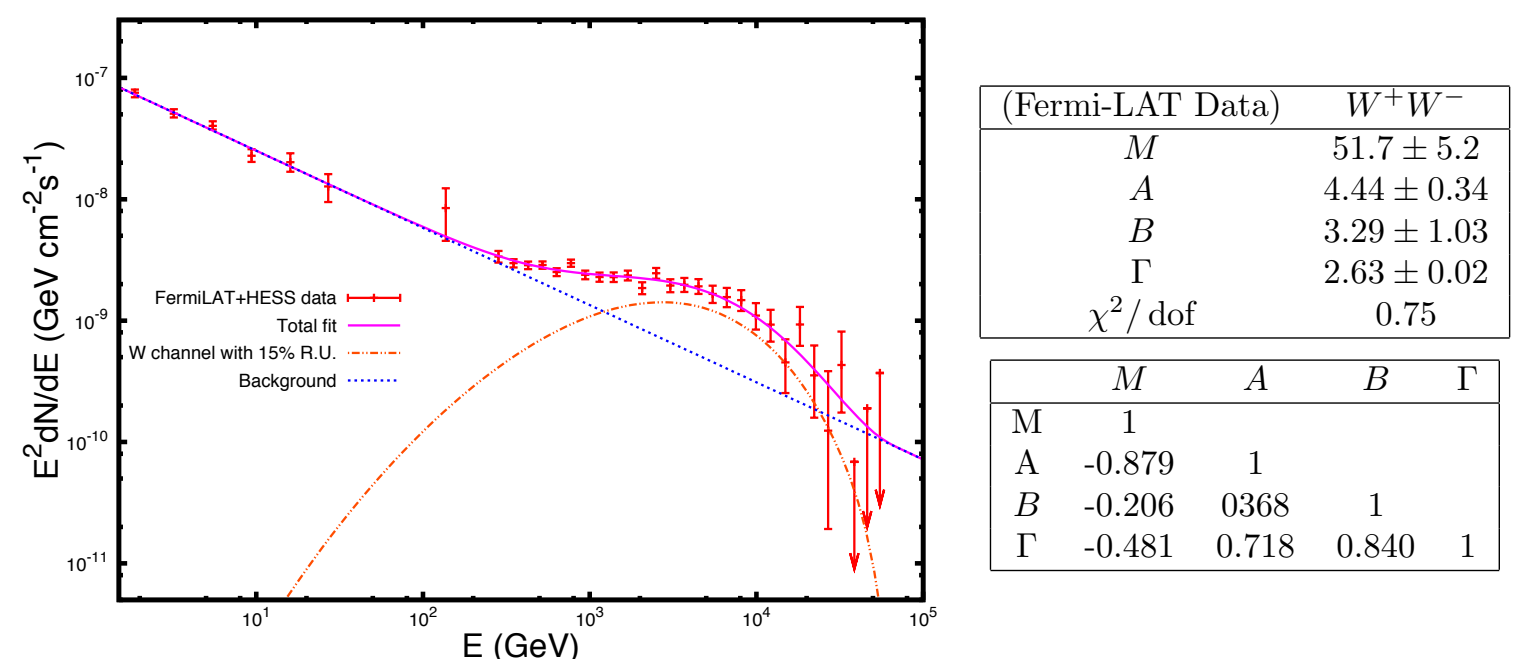

Figure 14. The same as in figure 12 but taking into account the Fermi-LAT data between $2 \mathrm{GeV} \lesssim$ $E \lesssim 100 \mathrm{GeV}$ [9], that extends the dof to 27 . The background parameters fitted by these data are $A=$ $(4.44 \pm 0.34) \cdot 10^{-7} \mathrm{~cm}^{-1} \mathrm{~s}^{-1 / 2}$ and $\Gamma=2.63 \pm 0.02$, in agreement with the spectral index for this spectral region already found when the Fermi-LAT data are fitted with a broken power law [9]. The quality of the fit is evident with a $\chi^{2} / d o f=0.75$. From this interpretation of the data, Fermi-LAT instruments are sensitive just to the background component of the signal.

contradiction with the unitarity limit for thermal relic annihilation. For example, this limit is around $110 \mathrm{TeV}$ for scalar DM particles annihilating in s-wave (for $\Omega_{\mathrm{DM}} h^{2}=0.11$ ) $[15,28]$. On the contrary, these heavy DM particles are practically unconstrained by direct detection experiments or particle colliders [29]. An interesting example of such type of DM candidates which could have high enough mass and account for the right amount of DM in the form of a thermal relic, is the branon $[30,31]$. Branons are new degrees of freedom corresponding to brane fluctuations in brane-world models. In general, they are natural candidates for DM because they are massive fields weakly interacting with the SM particles, since their interaction is suppressed with the fourth power of the brane tension scale $f$. For masses 


\begin{tabular}{|c|c|}
\hline Parameter & Fitting power law(s) \\
\hline$p$ & $4530 M^{0.653}$ \\
\hline$q$ & $0.00230 M^{-0.911}+0.00291 M^{0.0348}$ \\
\hline$l$ & $0.626 M^{-0.0300}+16.4 M^{-1.34}$ \\
\hline
\end{tabular}

Table 1. Mass dependent parameters for the fitting function of the photon spectrum coming from the $\mu^{+} \mu^{-}$channel.

\begin{tabular}{|c|c|c|c|c|c|c|c|c|c|}
\hline & \multicolumn{2}{|c|}{ Parameter } & \multicolumn{6}{|c|}{ Fitting power law } & \\
\hline & \multirow{2}{*}{\multicolumn{2}{|c|}{$n_{1}$}} & \multicolumn{6}{|c|}{$-7.00 M^{-1.99}+179 M^{-0.763}+9.09$} & \\
\hline & & & \multicolumn{6}{|c|}{$3.07 M^{1.55}$} & \\
\hline Channel & $a_{1}$ & $b_{1}$ & $b_{2}$ & $n_{2}$ & $c_{1}$ & $d_{1}$ & $c_{2}$ & $d_{2}$ & $q$ \\
\hline$\tau$ & 14.7 & 5.40 & 5.31 & 1.40 & 2.54 & 0.295 & 0.373 & 0.470 & 0.00260 \\
\hline
\end{tabular}

Table 2. Mass dependent and independent parameters for the $\tau^{+} \tau^{-}$channel fitting function.

\begin{tabular}{|c|c|c|c|c|c|c|c|}
\hline Parameter & Fitting power law & & & & & & \\
\hline$b_{1}$ & $2.96 M^{0.0506}$ & & & & & & \\
\hline$n_{1}$ & $2.91 M^{-0.351}+1.90 M^{0.0172}$ & Channel & $a_{1}$ & $b_{2}$ & $c_{1}$ & $c_{2}$ & $q$ \\
\hline$n_{2}$ & $0.0587 M^{0.146}+0.848 M^{-0.145}$ & $u \bar{u}$ & 5.58 & 5.50 & 0.315 & 0.0 & $9.30 \cdot 10^{-4}$ \\
\hline$d_{1}$ & $0.317 M^{-0.0300}+0.403 M^{-0.351}$ & & & & & & \\
\hline$p$ & $4.74 M^{0.839}$ & & & & & & \\
\hline
\end{tabular}

Table 3. Parameters for the $u \bar{u}$ channel fitting function.

over $100 \mathrm{GeV}$, the main contribution to the photon spectra comes from branons annihilating into gauge bosons $Z Z$, and $W^{+} W^{-}$. In [15] it was shown that a branon DM with mass $M \simeq 50.6 \mathrm{TeV}$, provides an excellent fit to HESS data. The corresponding background being compatible with Fermi-LAT data. The compatibility of its thermal abundance with the WMAP constraints [32], demands a cross section of $\langle\sigma v\rangle=(1.14 \pm 0.19) \cdot 10^{-26} \mathrm{~cm}^{3} \mathrm{~s}^{-1}$, what is equivalent to a brane tension of $f \simeq 27.5 \mathrm{TeV}$.

The analysis of other cosmic rays [33] from the GC and from other astrophysical objects is fundamental to cross check the hypotheses considered in this work. Updated analyses of this kind of signals for heavy DM combined with simple background components would be of great interest in order to constrain the possible DM origin of the studied gamma-ray fluxes.

\section{A Fitting function parameters}

In the following tables, we show explicitly the parameters used in the fitting functions. The precision associated with them are better than $10 \%$. An extrapolation of PYTHIA results is necessary for high energies. This fact is justified since the mass dependence of the fitting parameters reaches an asymptotic behavior for energies higher than $1 \mathrm{TeV}$ depending on the particular channel and parameter (read [26] for further details). 


\begin{tabular}{|c|c||c|c|c|c|c|c|}
\hline Parameter & \multicolumn{2}{|c|}{ Fitting power law } & \multicolumn{3}{|l|}{} \\
\hline$b_{1}$ & $3.39 M^{0.0485}$ & Channel & $a_{1}$ & $b_{2}$ & $d_{1}$ & $c_{2}$ & $q$ \\
\hline$n_{1}$ & $21.8 M^{-0.993}+2.25 M^{-0.00113}$ \\
\hline$n_{2}$ & $0.848 M^{-0219}+0.161 M^{0.0573}$ \\
\hline$c_{1}$ & $0.722 M^{-0.270}+0.0544 M^{0.0874}$ \\
\hline$p$ & $0.168 M^{1.29}$ \\
\hline
\end{tabular}

Table 4. The analogous set of parameters shown in table 3 but for the $d \bar{d}$ channel.

\begin{tabular}{|c|c||c|c|c|c|c|c|c|}
\hline Parameter & \multicolumn{2}{|c|}{ Fitting power law } \\
\hline$b_{1}$ & $4.54 M^{0.0339}$ \\
\hline$n_{2}$ & $3.68 M^{-1.01}+0.744 M^{-0.0352}$ \\
\hline$d_{1}$ & $0.621 M^{-0.674}+0.414 M^{-0.0588}$ \\
\hline$p$ & $12.8 M^{0.732}$ \\
\hline$p$ & $s \bar{s}$ & 4.83 & 2.03 & 6.50 & 0.335 & $2.40 \cdot 10^{-4}$ \\
\hline
\end{tabular}

Table 5. Parameters for the fitting function of the photon spectrum coming from DM annihilation in $s \bar{s}$ channel.

\begin{tabular}{|c|c|c|c|c|c|c|c|}
\hline Parameter & Fitting power law & & & & & & \\
\hline$b_{1}$ & $9.90 M^{-0.130}$ & & & & & & \\
\hline$n_{1}$ & $4.14 M^{-0.148}$ & Channel & $a_{1}$ & $b_{2}$ & $n_{2}$ & $c_{2}$ & $q$ \\
\hline$c_{1}$ & $0.210 M^{0.0951}$ & $c \bar{c}$ & 5.58 & 7.90 & 0.686 & 0.0 & $9.00 \cdot 10^{-4}$ \\
\hline$d_{1}$ & $1.50 M^{-0.632}+0.479 M^{-0.0942}$ & & & & & & \\
\hline$p$ & $8.11 M^{0.812}$ & & & & & & \\
\hline
\end{tabular}

Table 6. The same as in table 3 for the $c \bar{c}$ channel.

\begin{tabular}{|c|c|c|c|c|c|c|c|}
\hline Parameter & Fitting power law & \multicolumn{3}{|c|}{} \\
\hline$b_{1}$ & $152 M^{-0.462}$ & \multicolumn{1}{|c|}{} \\
\hline$n_{1}$ & $18.7 M^{-0.248}$ \\
\hline$n_{2}$ & $0.707 M^{-0.0129}$ \\
\hline$c_{1}$ & $0.328 M^{0.0447}$ \\
\hline$d_{1}$ & $0.449 M^{-0.0552}$ \\
\hline$p$ & $11.8 M^{0.641}$ \\
\hline$b$ & & & $a_{1}$ & $b_{2}$ & $c_{2}$ & $d_{2}$ & $q$ \\
\hline & & & 10.0 & 11.0 & 0.0151 & 0.550 & $2.60 \cdot 10^{-4}$ \\
\hline
\end{tabular}

Table 7. Parameters for the $b \bar{b}$ channel fitting function.

\section{Acknowledgments}

We thank Alvaro de la Cruz-Dombriz for useful comments. This work has been supported by MICINN (Spain) This work has been supported by MICINN (Spain) project numbers FIS 2008-01323, FIS2011-23000, FPA2011-27853-01 and Consolider-Ingenio MULTIDARK CSD2009-00064. 


\begin{tabular}{|c|c|c|c|c|c|c|}
\hline Parameter & Fitting power law(s) & \multirow{5}{*}{$\frac{\text { Channel }}{t \bar{t}}$} & \multirow{4}{*}{$a_{1}$} & \multirow{4}{*}{$c_{1}$} & \multirow{4}{*}{$d_{1}$} & \multirow{4}{*}{$d_{2}$} \\
\hline$b_{1}$ & $16.4 M^{-0.0400}$ & & & & & \\
\hline$n_{1}$ & $0.559 M^{-0.0379}$ & & & & & \\
\hline$c_{2}$ & $8910 M^{-3.23}$ & & & & & \\
\hline$p$ & $5.78 \cdot 10^{-5} M^{1.89}$ & & 290 & 1.61 & 0.19 & 0.845 \\
\hline$q$ & $0.133 M^{0.488}$ & & & & & \\
\hline$l$ & $21.9 M^{-0.302}$ & & & & & \\
\hline
\end{tabular}

Table 8. Mass depend and independent parameter of the fitting function given by eq. (4.3) for the $t \bar{t}$ channel.

\begin{tabular}{|c|c|c|c|}
\hline Channel & $a_{1}$ & $n_{1}$ & $q$ \\
\hline $\mathrm{W}$ & 25.8 & 0.510 & 3.00 \\
\hline $\mathrm{Z}$ & 25.8 & 0.5 & 3.87 \\
\hline
\end{tabular}

\begin{tabular}{|c|c|c|c|}
\hline Channel & $b_{1}$ & $c_{1}$ & $d_{1}$ \\
\hline$W^{+} W^{-}$ & $9.29 M^{-0.0139}$ & $0.743 M^{0.0331}$ & $0.265 M^{-0.0137}$ \\
\hline$Z Z$ & $9.36 M^{-0.00710}$ & $0.765 M^{0.00980}$ & $0.272 M^{-0.00990}$ \\
\hline
\end{tabular}

\begin{tabular}{|c|c|c|}
\hline Channel & $p$ & $j$ \\
\hline$W^{+} W^{-}$ & $10^{5} M^{-1.13}+285 M^{0.0794}$ & $0.943 M^{0.00852}$ \\
\hline$Z Z$ & $85505 M^{-0.166}+0.476 M^{0.984}$ & $0.884 M^{0.0175}$ \\
\hline
\end{tabular}

Table 9. Parameters for the fitting functions of the electroweak gauge bosons channels.

\section{References}

[1] MAGIC collaboration, J. Aleksic et al., Searches for Dark Matter annihilation signatures in the Segue 1 satellite galaxy with the MAGIC-I telescope, JCAP 06 (2011) 035 [arXiv: 1103.0477] [INSPIRE].

[2] Fermi-LAT collaboration, A. Abdo et al., Observations of Milky Way Dwarf Spheroidal galaxies with the Fermi-LAT detector and constraints on Dark Matter models, Astrophys. J. 712 (2010) 147 [arXiv: 1001.4531] [INSPIRE].

[3] CANGAROO-II collaboration, K. Tsuchiya et al., Detection of sub-TeV gamma-rays from the Galactic Center direction by CANGAROO-II, Astrophys. J. 606 (2004) L115 [astro-ph/0403592] [INSPIRE].

[4] VERITAS collaboration, K. Kosack et al., TeV gamma-ray observations of the galactic center, Astrophys. J. 608 (2004) L97 [astro-ph/0403422] [INSPIRE].

[5] HESS collaboration, F. Aharonian et al., Very high-energy gamma rays from the direction of Sagittarius A*, Astron. Astrophys. 425 (2004) L13 [astro-ph/0408145] [INSPIRE].

[6] HESS collaboration, F. Aharonian and F. Aharonian, Spectrum and variability of the Galactic Center VHE gamma-ray source HESS J1745-290, Astron. Astrophys. 503 (2009) 817 [arXiv: 0906.1247] [INSPIRE].

[7] MAGIC collaboration, J. Albert et al., Observation of gamma-rays from the galactic center with the magic telescope, Astrophys. J. 638 (2006) L101 [astro-ph/0512469] [INSPIRE]. 
[8] Fermi/LAT collaboration, V. Vitale and A. Morselli, Indirect Search for Dark Matter from the center of the Milky Way with the Fermi-Large Area Telescope, arXiv:0912.3828 [INSPIRE].

[9] M. Chernyakova, D. Malyshev, F. Aharonian, R. Crocker and D. Jones, The high-energy, Arcminute-scale galactic center gamma-ray source, Astrophys. J. 726 (2011) 60 [arXiv: 1009.2630] [INSPIRE];

T. Linden, E. Lovegrove and S. Profumo, The Morphology of Hadronic Emission Models for the Gamma-Ray Source at the Galactic Center, Astrophys. J. 753 (2012) 41 [arXiv:1203.3539] [INSPIRE].

[10] D. Horns, TeV gamma-radiation from dark matter annihilation in the Galactic center, Phys. Lett. B 607 (2005) 225 [Erratum ibid. B 611 (2005) 297] [astro-ph/0408192] [INSPIRE].

[11] L. Bergstrom, T. Bringmann, M. Eriksson and M. Gustafsson, Gamma rays from Kaluza-Klein dark matter, Phys. Rev. Lett. 94 (2005) 131301 [astro-ph/0410359] [InSPIRE]; Gamma rays from heavy neutralino dark matter, Phys. Rev. Lett. 95 (2005) 241301 [hep-ph/0507229] [INSPIRE].

[12] S. Profumo, TeV gamma-rays and the largest masses and annihilation cross sections of neutralino dark matter, Phys. Rev. D 72 (2005) 103521 [astro-ph/0508628] [INSPIRE].

[13] HESS collaboration, F. Aharonian et al., H.E.S.S. observations of the Galactic Center region and their possible dark matter interpretation, Phys. Rev. Lett. 97 (2006) 221102 [Erratum ibid. 97 (2006) 249901] [astro-ph/0610509] [INSPIRE].

[14] F. Aharonian and A. Neronov, High energy gamma rays from the massive black hole in the Galactic center, Astrophys. J. 619 (2005) 306 [astro-ph/0408303] [INSPIRE].

[15] J. Cembranos, V. Gammaldi and A. Maroto, Possible dark matter origin of the gamma ray emission from the galactic center observed by HESS, Phys. Rev. D 86 (2012) 103506 [arXiv: 1204.0655] [INSPIRE].

[16] A.V. Belikov, G. Zaharijas and J. Silk, Study of the Gamma-ray Spectrum from the Galactic Center in view of Multi-TeV Dark Matter Candidates, Phys. Rev. D 86 (2012) 083516 [arXiv: 1207.2412] [INSPIRE].

[17] R.M. Crocker, M. Fatuzzo, R. Jokipii, F. Melia and R.R. Volkas, The AGASA/SUGAR anisotropies and TeV gamma rays from the Galactic Center: A Possible signature of extremely high-energy neutrons, Astrophys. J. 622 (2005) 892 [astro-ph/0408183] [INSPIRE].

[18] Q.D. Wang, F. Lu and E. Gotthelf, G359.95-0.04: pulsar candidate near sgr a*, Mon. Not. Roy. Astron. Soc. 367 (2006) 937 [astro-ph/0512643] [INSPIRE];

B. Aschenbach, N. Grosso, D. Porquet and P. Predehl, X-ray flares reveal mass and angular momentum of the Galactic Center black hole, Astron. Astrophys. 417 (2004) 71 [astro-ph/0401589] [INSPIRE].

[19] A. Atoyan and C.D. Dermer, TeV emission from the Galactic Center black-hole plerion, Astrophys. J. 617 (2004) L123 [astro-ph/0410243] [INSPIRE].

[20] G.R. Blumenthal, S. Faber, R. Flores and J.R. Primack, Contraction of Dark Matter Galactic Halos Due to Baryonic Infall, Astrophys. J. 301 (1986) 27 [INSPIRE];

O.Y. Gnedin, A.V. Kravtsov, A.A. Klypin and D. Nagai, Response of dark matter halos to condensation of baryons: Cosmological simulations and improved adiabatic contraction model, Astrophys. J. 616 (2004) 16 [astro-ph/0406247] [INSPIRE].

[21] F. Prada, A. Klypin, J. Flix Molina, M. Martínez and E. Simonneau, Dark Matter Annihilation in the Milky Way Galaxy: Effects of Baryonic Compression, Phys. Rev. Lett. 93 (2004) 241301 [astro-ph/0401512] [INSPIRE].

[22] E. Romano-Díaz, I. Shlosman, Y. Hoffman and C. Heller, Erasing Dark Matter Cusps in Cosmological Galactic Halos with Baryons, Astrophys. J. Lett. 685 (2008) L105 [arXiv:0808.0195]; 
E. Romano-Díaz, I. Shlosman, C. Heller and Y. Hoffman, Dissecting Galaxy Formation: I. Comparison Between Pure Dark Matter and Baryonic Models, Astrophys. J. 702 (2009) 1250 [arXiv:0901.1317] [InSPIRE];

A.V. Maccio', G. Stinson, C.B. Brook, J. Wadsley, H. Couchman et al., Halo expansion in cosmological hydro simulations: towards a baryonic solution of the cusp/core problem in massive spirals, arXiv:1111.5620 [INSPIRE].

[23] P. Salucci, M.I. Wilkinson, M.G. Walker, G.F. Gilmore, E.K. Grebel et al., Dwarf spheroidal galaxy kinematics and spiral galaxy scaling laws, arXiv:1111.1165 [INSPIRE].

[24] T. Sjöstrand, S. Mrenna and P.Z. Skands, PYTHIA 6.4 Physics and Manual, JHEP 05 (2006) 026 [hep-ph/0603175] [INSPIRE].

[25] J.F. Navarro, C.S. Frenk and S.D. White, A Universal density profile from hierarchical clustering, Astrophys. J. 490 (1997) 493 [astro-ph/9611107] [InSPIRE].

[26] J. Cembranos, A. de la Cruz-Dombriz, A. Dobado, R. Lineros and A. Maroto, Photon spectra from WIMP annihilation, Phys. Rev. D 83 (2011) 083507 [arXiv:1009.4936] [InSPIRE]; Photon spectra from quark generation by WIMPs, AIP Conf. Proc. 1343 (2011) 595 [arXiv: 1011.2137] [INSPIRE]; Fitting formulae for photon spectra from WIMP annihilation, J. Phys. Conf. Ser. 314 (2011) 012063 [arXiv: 1012.4473] [INSPIRE];

A. de la Cruz-Dombriz and V. Gammaldi, Dark Matter with Photons, arXiv:1109.5027 [INSPIRE]; http://teorica.fis.ucm.es/PaginaWeb/photon_spectra.html.

[27] J. Cohen-Tanugi, M. Pohl, O. Tibolla and E. Nuss, The GeV-band source population in the Galactic-Center region as seen by Fermi Large Area Telescope, in Proc. 31st ICRC (Lodz) (2009) 645.

[28] K. Griest and M. Kamionkowski, Unitarity Limits on the Mass and Radius of Dark Matter Particles, Phys. Rev. Lett. 64 (1990) 615 [inSPIRE].

[29] J. Alcaraz, J. Cembranos, A. Dobado and A.L. Maroto, Limits on the brane fluctuations mass and on the brane tension scale from electron positron colliders, Phys. Rev. D 67 (2003) 075010 [hep-ph/0212269] [INSPIRE];

L3 collaboration, P. Achard et al., Search for branons at LEP, Phys. Lett. B 597 (2004) 145 [hep-ex/0407017] [INSPIRE];

J. Cembranos, A. Dobado and A.L. Maroto, Brane skyrmions and wrapped states, Phys. Rev. D 65 (2002) 026005 [hep-ph/0106322] [InSPIRE]; Branon search in hadronic colliders, Phys. Rev. D 70 (2004) 096001 [hep-ph/0405286] [InSPIRE]; Branon radiative corrections to collider physics and precision observables, Phys. Rev. D 73 (2006) 035008 [hep-ph/0510399] [INSPIRE]; Dark matter clues in the muon anomalous magnetic moment, Phys. Rev. D 73 (2006) 057303 [hep-ph/0507066] [INSPIRE]; Some model-independent phenomenological consequences of flexible brane worlds, J. Phys. A 40 (2007) 6631 [hep-ph/0611024] [InSPIRE]. J.A. Cembranos, J.L. Diaz-Cruz and L. Prado, Impact of DM direct searches and the LHC analyses on branon phenomenology, Phys. Rev. D 84 (2011) 083522 [arXiv:1110.0542] [InSPIRE].

[30] A. Dobado and A.L. Maroto, The Dynamics of the Goldstone bosons on the brane, Nucl. Phys. B 592 (2001) 203 [hep-ph/0007100] [INSPIRE];

J. Cembranos, A. Dobado and A.L. Maroto, Brane world dark matter, Phys. Rev. Lett. 90 (2003) 241301 [hep-ph/0302041] [INSPIRE]; Cosmological and astrophysical limits on brane fluctuations, Phys. Rev. D 68 (2003) 103505 [hep-ph/0307062] [INSPIRE];

A.L. Maroto, The Nature of branon dark matter, Phys. Rev. D 69 (2004) 043509 [hep-ph/0310272] [INSPIRE]; Brane oscillations and the cosmic coincidence problem, Phys. Rev. D 69 (2004) 101304 [hep-ph/0402278] [INSPIRE];

J. Cembranos, A. Dobado and A.L. Maroto, Dark geometry, Int. J. Mod. Phys. D 13 (2004) 2275 [hep-ph/0405165] [INSPIRE]; 
J. Cembranos, A. de la Cruz-Dombriz, A. Dobado and A.L. Maroto, Is the CMB Cold Spot a gate to extra dimensions?, JCAP 10 (2008) 039 [arXiv:0803.0694] [INSPIRE].

[31] J. Cembranos, A. de la Cruz-Dombriz, V. Gammaldi and A. Maroto, Detection of branon dark matter with gamma ray telescopes, Phys. Rev. D 85 (2012) 043505 [arXiv:1111.4448] [INSPIRE].

[32] WMAP collaboration, E. Komatsu et al., Seven-Year Wilkinson Microwave Anisotropy Probe (WMAP) Observations: Cosmological Interpretation, Astrophys. J. Suppl. 192 (2011) 18 [arXiv: 1001.4538 ] [INSPIRE].

[33] S. Rudaz and F. Stecker, Cosmic ray anti-protons, positrons and gamma-rays from halo dark matter annihilation, Astrophys. J. 325 (1988) 16 [INSPIRE];

J.A. Cembranos, J.L. Feng, A. Rajaraman and F. Takayama, SuperWIMP solutions to small scale structure problems, Phys. Rev. Lett. 95 (2005) 181301 [hep-ph/0507150] [INSPIRE];

J.A. Cembranos, J.L. Feng and L.E. Strigari, Resolving Cosmic Gamma Ray Anomalies with Dark Matter Decaying Now, Phys. Rev. Lett. 99 (2007) 191301 [arXiv:0704.1658] [InSPIRE]; Exotic Collider Signals from the Complete Phase Diagram of Minimal Universal Extra Dimensions, Phys. Rev. D 75 (2007) 036004 [hep-ph/0612157] [INSPIRE];

J.A. Cembranos and L.E. Strigari, Diffuse MeV Gamma-rays and Galactic $511 \mathrm{keV}$ Line from Decaying WIMP Dark Matter, Phys. Rev. D 77 (2008) 123519 [arXiv:0801.0630] [INSPIRE]; J.A. Cembranos, Dark Matter from R2-gravity, Phys. Rev. Lett. 102 (2009) 141301 [arXiv:0809.1653] [inSPIRE]; The Newtonian limit at intermediate energies, Phys. Rev. D 73 (2006) 064029 [gr-qc/0507039] [INSPIRE];

T. Bringmann and C. Weniger, Gamma Ray Signals from Dark Matter: Concepts, Status and Prospects, Phys. Dark Univ. 1 (2012) 194 [arXiv:1208.5481] [INSPIRE]. 